\title{
Research Paper: The Effect of Voluntary and Elec- trically Stimulated Quadriceps Muscle Fatigue on Postural Control
}

\author{
Mostafa Sirousi $^{1}$ (D), Mohammad Akbari ${ }^{1}$ (D), Zahra Teymuri²* ${ }^{*}$, Asghar Akbari ${ }^{2,3}$ (D) \\ 1. Department of Physiotherapy, School of Rehabilitation Sciences, Iran University of Medical Sciences, Tehran, Iran. \\ 2. Department of Physiotherapy, Faculty of Rehabilitation Sciences, Zahedan University of Medical Sciences, Zahedan, Iran. \\ 3. Health Promotion Research Center, Zahedan University of Medical Sciences, Zahedan, Iran.
}

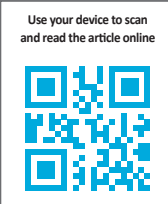

chtation Sirousi M, Akbari M, Teymuri Z, Akbari A. The Effect of Voluntary and Electrically Stimulated Quadriceps Muscle Fatigue on Postural Control. Physical Treatments. 2018; 7(4):225-232. http://dx.doi.org/10.32598/ptj.7.4.225

doi : http://dx.doi.org/10.32598/ptj.7.4.225

Funding: See Page 231

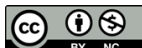

Article info:

Received: 25 Jun 2017

Accepted: 13 Oct 2017

Available Online: 01 Jan 2018

Keywords:

Postural control, Quadriceps fatigue, Voluntary contraction, Electrical Stimulation

\section{A B S T RA C T}

Purpose: The present study aimed to investigate the effect of voluntary and electrically stimulated quadriceps muscle fatigue on postural control.

Methods: Sixteen healthy young males participated in this quasi-experimental study. All subjects performed two fatiguing exercises with equal intensity of isometric maximal voluntary contraction: Voluntary (VOL) and Electrical Stimulation (ES). The Centre of Pressure (CoP) displacement in the VOL and ES exercises were measured before and after conducting each protocol. The CoP displacement variables were analyzed by repeated measures ANOVA in SPSS. The Newman-Keuls post hoc test was used to determine the mean score differences.

Results: The VOL and ES fatiguing exercises had significant effects on mean scores of sway area, mean velocity, anteroposterior, and mediolateral displacements. There was a significant difference between VOL and ES fatigue on postural control $(\mathrm{P}<0.05)$.

Conclusion: The VOL fatiguing exercise impaired postural control more significantly than the ES fatiguing exercise. The disturbance of postural control in quadriceps muscle fatigue is related to the nature of the muscle contractions rather than the magnitude of strength loss.

\footnotetext{
* Corresponding Author:

Zahra Teymuri, MSc.

Address: Department of Physiotherapy, Faculty of Rehabilitation Sciences, Zahedan University of Medical Sciences, Zahedan, Iran.

Phone: +98 (56) 32239917

E-mail: z.teymuri.pt@gmail.com
} 


\section{Highlights}

- Quadriceps muscle has an important action or function in maintaining unipedal postural control.

- Either of Electrical stimulation and Voluntary contractions of this muscle can lead to its fatigue and then postural control disturbance.

- It might not be necessary and needful to train this muscle with to kind of this exercises simultaneously in one session.

- In initial sessions may be its enough to just train the quadriceps muscle with the Electrical Stimulation.

\section{Plain Language Summary}

Postural control means that we can hold ourselves on one or two legs steady without falling. For this hold, one of the important muscle that help us is quadriceps muscle. It needs to be strong but sometimes when its getting fatigue like when we exercise or doing a hard work without rest it might lead to a situation that we can not control our posture and cause falling. This study showed that just strength loss is not the only factor that make disturbance of postural control. Quadriceps muscle has two kind of fibers: Slow fibers and Fast fibers. If the work that we do be a kind that engages the slow fibers more than the fast fibers the risk of falling getting increased and it might be necessary to be careful about Quadriceps muscle fatigue.

\section{Introduction}

ostural control is the ability to maintain body position within the base of support [1]. The Central Nervous System (CNS) receives and integrates sensory and motor information to handle postural control. Manipulation of sensory-motor information affects the integrated information and impairs postural control [2-4].

Decreased force-generating capacity of the muscle at the level of neuromuscular junction or distal to it is defined as fatigue that is an important factor in postural control impairment [5]. Fatigue can lead to an acute impairment in the neuromuscular system by changes in muscle strength and neuromuscular control. These changes increase Centre of Pressure $(\mathrm{CoP})$ displacement and affect postural control [6]. Repetitive muscular contractions can induce localized muscle fatigue [7]. Therefore, the voluntary and electrically contractions decrease muscle strength and generate fatigue [8-10]. The different levels of acute muscle strength loss cause various degrees of postural control disturbance [11].

The Voluntary (VOL) and Electrical Stimulation (ES) contractions have different physiological impacts on muscles [12]. The ES fatigue compared to VOL fatigue produce more soreness [13] and cytoplasm acidity [14]. These changes can alter proprioceptive mechanism and impair postural control in the ES more than the VOL fatigue [10]. Paillard and Vanderthommen reported that the level of muscle strength loss are not equal after VOL and ES fatiguing exercises, under the similar intensity and duration of quadriceps muscle contractions [14-16].

A review study by Paillard reported that $25 \%$ to $30 \%$ of Maximal Voluntary Contraction (MVC) loss can affect postural control [15]. To prevent painful muscle contractions in ES exercise, the minimal amount of MVC loss $(30 \%)$ is used. The current study aimed to compare the effect of stimulated versus voluntary contractions on ipsilateral unipedal postural control. Previous studies reported different decrease in MVC in voluntary and electrical stimulation of quadriceps muscle. The VOL and ES contractions are different. The voluntary contractions first recruit the small motor neurons while in the ES contractions the large motor neurons are initially activated $[17,18]$. Previous studies reported the effects of two fatiguing exercises on postural control conducted in an equal duration $[10,15,16,19,20]$.

The present study was conducted to address the literature gap on the effects of equal amount of strength loss on postural control. We hypothesized that with equal strength loss, VOL fatigue could disturb postural control more than ES fatigue. 


\section{Materials and Methods}

\section{Study subjects}

In this quasi-experimental study, 16 healthy young males participated, between November 2016 and April 2017. In all subjects, right leg was the dominant leg (the dominant leg is used for kicking a ball). The inclusion criteria comprised no history of neurological or musculoskeletal impairment in the past two years, no balance disorders or any documented postural control disorders, no medical condition that might affect postural control, no disinclination of participants before or during the test, no limb mal-alignment (pronated or supinated foot, knee hyperextension, etc.), and no affecting factor or current injury making the subject unable to participate in the research (ankle sprain, knee trauma, etc.). All participants signed an informed consent form approved by the local Ethics Committee. Subjects were asked to avoid any strenuous activity or movement, two hours before exercise session.

The fatiguing exercises were performed in random order by each subject. The study comprised two different fatiguing exercises; voluntary contractions of the quadriceps muscle (VOL exercise) and Electrical Stimulation of the quadriceps muscle (ES exercise). In order to assess the possible postural changes on the ipsilateral limb in the unipedal stance, the isometric MVC was measured before (pre-fatigue or pretest) and immediately after (post-fatigue or posttest) each exercise completion.

\section{Study apparatus}

A force plate (Kistler-9260AA6, $4^{\text {th }}$ order, $10 \mathrm{~Hz}$ lowpass Butterworth filter, the data were sampled at 100 $\mathrm{Hz}$ ), digital dynamometer (Commander Power Track II HHD. J Tech Medical, USA), portable electrical stimulator delivering a maximum current intensity of 160 V (Stimulator 620 P Version: 01, Tehran, Iran) with 4 rectangular rubber electrodes (size $4 \times 6 \mathrm{~cm}$, Iran) were used for postural control recording, measuring and controlling of the pre- and post-MVC and the ES fatiguing exercise, respectively.

\section{Study procedure}

Unilateral postural control was analysed before (pretest) and immediately after (posttest) the two exercises. The subjects were asked to stand on the dominant leg while the big toe of non-dominant leg lightly touched the medial malleolus of the supporting leg. The $\mathrm{X}$ and $\mathrm{Y}$ axes were glued to the force plate. The study proce- dure was explained to the participants. At first, the subjects were asked to perform 15 minutes of warm-up on a cycle ergometer at low intensity. Then, they stood on force plate for 30 seconds according to precise $\mathrm{X}$ and $\mathrm{Y}$ axes, barefoot, and as immobile as possible to measure pre-fatigue control.

The subjects were asked to sit on the edge of the fixed table with a $90^{\circ}$ of hip and knee flexion with arms crossed on the chest and perform the isometric MVC of dominant quadriceps muscle. A digital dynamometer was previously placed and fixed on distal portion of the tibia. Three MVCs, with 5-second contraction and 30 seconds of rest between each contraction were recorded by the digital dynamometer. Subjects were guided verbally about their performance.

The peak force (in $\mathrm{kg}$ ) served as a workload during the fatiguing exercise. After 30 minutes resting, the subjects began the fatiguing exercises in one session and in random order (VOL and ES exercises). After completing each fatiguing exercise, the dynamometer was removed and posttest postural control was performed, at the soonest possible. For quick recovery of neuromuscular system, two hours of rest were considered to obviate the effects of the first fatiguing exercise. The workload of each contraction was kept and controlled at the level of $20 \%$ MVC by the digital dynamometer.

\section{Voluntary fatigue protocol}

The voluntary isometric contractions with intensity of $20 \% \mathrm{MVC}$ were performed for $5 \mathrm{~s}$ with $2 \mathrm{~s}$ rest between each contraction. The workload of contractions was controlled in $20 \%$ MVC by the examiner's verbal feedback and the dynamometer. After each 15 or 20 contractions, an MVC test was demonstrated and as soon as strength loss reached $30 \%$ of MVC, post-postural control test was performed. The force plate was placed at 3-m distance from the subject.

\section{Induced electrical stimulation fatigue protocol}

Four electrodes were placed over 4 parts of the quadriceps muscle. One electrode was placed over the proximal part of quadriceps femoris muscle across the vastus lateralis and rectus femoris muscles and 3 electrodes over the motor point of the vastus medialis, vastus lateralis, and rectus femoris [19]. Electrical stimulation was set in biphasic symmetrical rectangular wave (continuous pulse $350 \mu \mathrm{s}$, frequency $80 \mathrm{~Hz}$, hold $5 \mathrm{~s}$, rest $2 \mathrm{~s}$ ). The intensity of stimulation was continuously adjusted to reach the level of $20 \% \mathrm{MVC}$ and was controlled by 
the dynamometer. After each 15 or 20 contractions, an MVC test was performed and as soon as strength loss reached the level of $30 \% \mathrm{MVC}$, post-postural control test was executed.

\section{Measurements}

Postural control parameters included sway area and mean velocity recorded by force plate. Signals from the force plate were sampled at $100 \mathrm{~Hz}$, and amplified and converted from analog to digital form through an $\mathrm{A} / \mathrm{D}$ converter. In all stages of postural control recordings, subject's eyes were closed with an eye cover to prevent vision from contributing to the regulation of postural control behaviours.

\section{Statistical analysis}

The body sway was computed through the Center of foot Pressure (COP) displacements. The parameters and dependent variations analyzed included the mean total sway area $\left(\mathrm{mm}^{2}\right)$, mean sway velocity $(\mathrm{mm} / \mathrm{s})$, anteroposterior $(\mathrm{mm})$ and mediolateral $(\mathrm{mm})$ displacements. The data were analyzed using SPSS. One-sample Kolmogorov-Smirnov test was used to assess the normal distribution of the numeric variables. COP displacement parameters (mean total sway area, mean sway velocity, anteroposterior, and mediolateral displacements) were analyzed using repeated measures ANOVA. Results were considered significant at $\mathrm{P}<0.05$. Newman-Keuls post hoc test used to determine the mean score differences.

\section{Results}

Multiple comparisons of dependent variations of postural control are presented in Tables 1, 2, 3, and 4. Both VOL and ES exercises had significant effects on the mean sway area, mean velocity, anteroposterior and mediolateral displacements $(\mathrm{P}<0.05)$. There was a significant difference between VOL and ES fatigue on postural control $(\mathrm{P}<0.05)$ (Tables 1, 2, 3, and 4). The VOL fatigue disturbed postural control more significantly than ES fatigue.

\section{Discussion}

This study investigated the effects of dominant quadriceps muscle voluntary and induced electrical stimulation fatigue on postural control. Ipsilateral unipedal postural control was disturbed after both VOL and ES fatigue

Table 1. Comparing total sway area before and after VOL and ES fatiguing exercises

\begin{tabular}{|c|c|c|c|c|c|c|}
\hline \multirow{2}{*}{ Dependent Variations } & & \multirow{2}{*}{ Group } & \multirow{2}{*}{$\begin{array}{c}\text { Mean } \\
\text { Difference }\end{array}$} & \multirow{2}{*}{$\mathbf{P}^{*}$} & \multicolumn{2}{|c|}{$0.95 \% \mathrm{Cl}$} \\
\hline & & & & & Lower Bound & Upper Bound \\
\hline \multirow{6}{*}{ Total sway area } & \multirow{2}{*}{$1^{*}$} & 2 & -1.69 & 0.001 & -9.40 & 6.03 \\
\hline & & 3 & 2.20 & 0.001 & -5.51 & 9.92 \\
\hline & \multirow{2}{*}{$2^{* *}$} & 1 & 1.69 & 0.001 & -6.03 & 9.40 \\
\hline & & 3 & 3.89 & 0.001 & -3.82 & 0.001 \\
\hline & \multirow{2}{*}{$3^{* * *}$} & 1 & -2.20 & 0.001 & -9.92 & 5.51 \\
\hline & & 2 & -3.89 & 0.001 & 0.001 & 3.82 \\
\hline
\end{tabular}

*:Before fatigue; **:After VOL fatigue; ***:After ES fatigue

PHYSICAL TREA MENTS

Table 2. Comparing multiple velocity sway before and after VOL and ES fatiguing exercises

\begin{tabular}{|c|c|c|c|c|c|c|}
\hline \multirow{2}{*}{ Dependent Variations } & \multirow{2}{*}{\multicolumn{2}{|c|}{ Group }} & \multirow{2}{*}{$\begin{array}{c}\text { Mean } \\
\text { Difference }\end{array}$} & \multirow{2}{*}{$\mathbf{P}$} & \multicolumn{2}{|c|}{$0.95 \% \mathrm{Cl}$} \\
\hline & & & & & Lower Bound & Upper Bound \\
\hline \multirow{9}{*}{ Velocity sway } & & 2 & -0.001 & 0.001 & 0.001 & 3.31 \\
\hline & $1^{*}$ & & & & & \\
\hline & & 3 & -0.001 & 0.001 & 0.001 & 3.16 \\
\hline & & 1 & 0.001 & 0.001 & 0.001 & 0.001 \\
\hline & $2^{* *}$ & & & & & \\
\hline & & 3 & 0.001 & 0.028 & 0.58 & 0.70 \\
\hline & & 1 & 0.001 & 0.001 & 0.001 & 0.001 \\
\hline & $3^{* * *}$ & & & & & \\
\hline & & 2 & -0.001 & 0.028 & -10.00 & 0.001 \\
\hline
\end{tabular}


Table 3. Comparing multiple anteroposterior displacement before and after VOL and ES fatiguing exercises

\begin{tabular}{|c|c|c|c|c|c|c|}
\hline \multirow{2}{*}{ Dependent Variations } & \multirow{2}{*}{\multicolumn{2}{|c|}{ Group }} & \multirow{2}{*}{$\begin{array}{c}\text { Mean } \\
\text { Difference }\end{array}$} & \multirow{2}{*}{$\mathbf{P}$} & \multicolumn{2}{|c|}{$0.95 \% \mathrm{Cl}$} \\
\hline & & & & & Lower Bound & Upper Bound \\
\hline \multirow{7}{*}{$\begin{array}{l}\text { Anteroposterior } \\
\text { displacement }\end{array}$} & \multirow{2}{*}{$1^{*}$} & 2 & -10.00 & 0.001 & -50.00 & 10.00 \\
\hline & & 3 & -0.001 & 0.001 & -30.00 & 20.00 \\
\hline & \multirow{2}{*}{$2^{* *}$} & 1 & 10.00 & 0.001 & -10.00 & 50.00 \\
\hline & & 3 & 10.00 & 0.001 & -10.00 & 40.00 \\
\hline & \multirow{3}{*}{$3^{* * *}$} & 1 & 0.001 & 0.001 & -20.00 & 30.00 \\
\hline & & & & & & \\
\hline & & 2 & -10.00 & 0.001 & -40.00 & 10.00 \\
\hline
\end{tabular}

Table 4. Comparing multiple mediolateral displacement before and after VOL and ES fatiguing exercises

\begin{tabular}{|c|c|c|c|c|c|c|}
\hline \multirow{2}{*}{ Dependent Variations } & & \multirow{2}{*}{ Group } & \multirow{2}{*}{$\begin{array}{c}\text { Mean } \\
\text { Difference }\end{array}$} & \multirow{2}{*}{$\mathbf{P}$} & \multicolumn{2}{|c|}{$0.95 \% \mathrm{Cl}$} \\
\hline & & & & & Lower Bound & Upper Bound \\
\hline \multirow{6}{*}{$\begin{array}{l}\text { Mediolateral } \\
\text { displacement }\end{array}$} & \multirow{2}{*}{$1^{*}$} & 2 & -10.00 & 0.001 & -40.20 & 0.001 \\
\hline & & 3 & -10.00 & 0.001 & -30.00 & 0.001 \\
\hline & \multirow{2}{*}{$2^{* *}$} & 1 & 10.00 & 0.001 & 0.001 & 40.20 \\
\hline & & 3 & 0.001 & 0.001 & -20.99 & 20.00 \\
\hline & \multirow{2}{*}{$3^{* * *}$} & 1 & 10.00 & 0.001 & 0.001 & 30.00 \\
\hline & & 2 & -0.001 & 0.001 & -20.00 & 20.99 \\
\hline
\end{tabular}

exercises. Evidence of ipsilateral quadriceps muscle fatigue was supported by the increased body sway area, velocity, $\mathrm{X}$ and $\mathrm{Y}$ displacements of the $\mathrm{COP}$ and by the $30 \%$ post MVC loss $[15,21]$. This study is the first description of equal VOL and ES fatigue on unipedal postural control. Previous studies indicate that VOL and ES fatigue disturb postural control in unipedal standing $[7$, $10,15,16,19,20,22,23]$.

The present results are more appealing because the MVC loss level was equal in both fatiguing exercises and can properly compare the effects of the 2 fatiguing exercises on postural control. Because the amount of MVC loss in both VOL and ES fatiguing contractions was equal, and postural control was differently affected, it can be assumed that muscle strength loss is not the only determining parameter in postural control disturbance. Accordingly, it can be concluded that the disturbance of the unipedal postural control after unilateral quadriceps muscle fatigue is especially related to the sensory input impairment.
Dicken and Doan (2008) reported that loss of muscle strength $(30 \%$ of $\mathrm{MVC})$ in knee muscles would lead to postural control disturbance [21]. A review by Paillard (2012) reported that $30 \%$ MVC or strength loss would impair postural control [15]. They also reported a relationship between the level of MVC loss and the velocity of the displacement of the Center of foot Pressure (CoP). About 30\% MVC loss in quadriceps muscle leads to postural control impairments [15]. Our study also confirmed that about $30 \%$ MVC loss in both VOL and ES fatiguing exercises disturbs postural control. Pline et al. reported that $40 \%$ MVC loss increase mean velocity, whereas mean velocity remains the same with $27 \%$ MVC loss [11].

Muscle fatigue by changes in limb central mapping representation [24], decreases muscle force generation capabilities [25-27] and proprioceptive deficit [28, 29] disturbs postural control. The present study indicates that VOL fatigue leads to greater postural control impairment than ES fatigue. The ES fatigue acidifies the cellular cytoplasm and reduces the intracellular $\mathrm{pH}$ more 


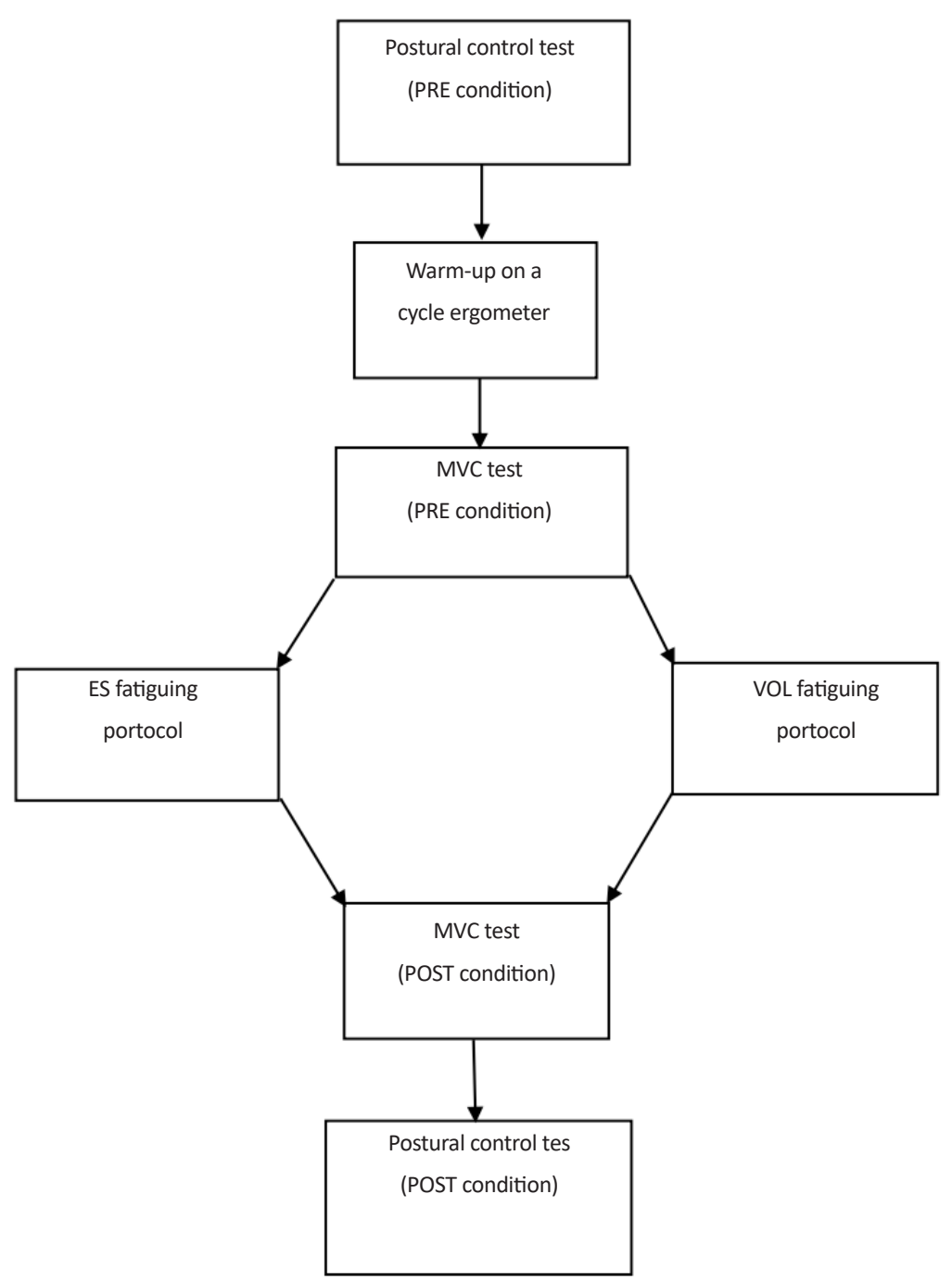

Figure 1. Chronological order of the fatigue protocols

The completion of each protocol (VOL and ES) was separated by 2 hours for all of the participants.

than VOL fatigue [30, 31]. With regard to the nature of muscle contraction, VOL contraction as a voluntary activation of muscle, is generated by central drive while the ES contraction as an artificial activation of muscle, is not generated by central drive [16].

Prolonged repetitive low-intensity contractions influence corticospinal output and declined output can lead to synaptic dysfunction [18, 32-34]. This phenomenon can affect the descending drives necessary for activation of motor neurons and influence movement control in the VOL fatigue [34]. In addition, according to the size recruitment principle in voluntary and electrical induced contractions [35], VOL contractions initially activate the small motor units located deep inside the muscle [30], while ES contractions first activate large motor units located on the quadriceps muscle surface [36].
VOL contractions induce more severe fatigue in the small fibres, which are mainly active in postural control, whereas ES contractions induces more severe fatigue in large fibres, with no specific role in postural control. The slow-twitch fibres (small fibres) control the standing posture especially in unipedal standing [37]. Therefore, VOL fatigue disturbs postural control more than ES fatigue. Moreover, we observed that in an equal MVC loss, reaching $30 \%$ of MVC loss takes longer time in VOL than ES fatigue. Prolonged VOL fatigue also leads to deficits in corticospinal output, and change in synaptic function $[17,18]$ that affect the movement control $[17,34]$ and has great effect on postural control than ES fatigue.

The present study also revealed that COP displacement in the anteroposterior direction Y, especially in the VOL fatigue is more than mediolateral direction $\mathrm{X}$. This find- 
ing indicates the specific role of quadriceps muscle function in knee joint stability at sagittal plane [9] more than frontal plane. These findings might also support studies that reported postural control impairment in quadriceps muscle [38, 39].

Both VOL and ES exercises induce fatigue in ipsilateral unipedal standing which disturb postural control. In addition, VOL fatigue disturbs postural control more than ES fatigue. The disturbance of postural control after quadriceps fatigue is mostly related to the nature of muscle contraction (voluntary vs. non-voluntary) than the magnitude of the strength loss. Future studies are recommended to investigate the effects of these fatiguing exercises on postural control in more than $30 \%$ level of fatigue (for example in 50\%).

These results may warn us of potential negative effects of voluntary or stimulated quadriceps muscle exercises in one session on postural control, especially in rehabilitation context. The current research also emphasizes the risk of falling after decreased muscle strength and loss of balance. It is important that clinicians do not use VOL and ES contractions to the extent that leads to fatigue.

\section{Ethical Considerations}

\section{Compliance with ethical guidelines}

All ethical principles were considered in this article. The participants were informed about the purpose of the research and its implementation stages; They were also assured about the confidentiality of their information; Moreover, They were allowed to leave the study whenever they wish, and if desired, the results of the research would be available to them.

\section{Funding}

This research has been supported by Iran University of Medical Sciences and the Health Services under the grant No: 320/682.

\section{Conflict of interest}

The authors declared no conflict of interest.

\section{Acknowledgements}

The authors thank all the participants for their helpful cooperation.

\section{References}

[1] Shumway-Cook A, Woollacott MH. Motor control: Theory and practical applications. Philadelphia: Williams \& Wilkins; 2005.

[2] Paillard T, Bizid R, Dupui P. Do sensorial manipulations affect subjects differently depending on their postural abilities. British Journal of Sports Medicine. 2007; 41(7):435-8. [DOI:10.1136/bjsm.2006.032904] [PMID] [PMCID]

[3] Popov KE, Kozhina GV, Smetanin BN, Shlikov VY. Postural responses to combined vestibular and hip proprioceptive stimulation in man. European Journal of Neuroscience. 1999; 11(9):3307-11. [DOI:10.1046/j.1460-9568.1999.00733.x] [PMID]

[4] Uimonen S, Sorri M, Laitakari K, Jämsä T. A comparison of three vibrators in static posturography: The effect of vibration amplitude on body sway. Medical Engineering \& Physics. 1996; 18(5):405-9. [DOI:10.1016/1350-4533(96)00079-3]

[5] Gribble PA, Hertel J. Effect of lower-extremity muscle fatigue on postural control 1. Archives of Physical Medicine and Rehabilitation. 2004; 85(4):589-92. [DOI:10.1016/j. apmr.2003.06.031] [PMID]

[6] Enoka RM, Stuart DG. Neurobiology of muscle fatigue. Journal of Applied Physiology. 1992; 72(5):1631-48. [DOI:10.1152/jappl.1992.72.5.1631] [PMID]

[7] Bizid R, Jully JL, Gonzalez G, François Y, Dupui P, Paillard T. Effects of fatigue induced by neuromuscular electrical stimulation on postural control. Journal of Science and Medicine in Sport. 2009; 12(1):60-6. [DOI:10.1016/j.jsams.2007.10.006] [PMID]

[8] Bisson EJ, McEwen D, Lajoie Y, Bilodeau M. Effects of ankle and hip muscle fatigue on postural sway and attentional demands during unipedal stance. Gait \& Posture. 2011; 33(1):83-7. [DOI:10.1016/j.gaitpost.2010.10.001] [PMID]

[9] Bizid R, Margnes E, François Y, Jully JL, Gonzalez G, Dupui $\mathrm{P}$, et al. Effects of knee and ankle muscle fatigue on postural control in the unipedal stance. European Journal of Applied Physiology. 2009; 106(3):375-80. [DOI:10.1007/s00421-0091029-2] [PMID]

[10] Paillard T, Margnes E, Maitre J, Chaubet V, François Y Jully JL, et al. Electrical stimulation superimposed onto voluntary muscular contraction reduces deterioration of both postural control and quadriceps femoris muscle strength Neuroscience. 2010; 165(4):1471-5. [DOI:10.1016/j.neuroscii ence.2009.11.052] [PMID]

[11] Pline KM, Madigan ML, Nussbaum MA. Influence of fatigue time and level on increases in postural sway. Ergonomics. 2006; 49(15):1639-48. [DOI:10.1080/00140130600901678] [PMID]

[12] Paillard T. Combined application of neuromuscular electrical stimulation and voluntary muscular contractions. Sports Medicine. 2008; 38(2):161-77. [DOI:10.2165/00007256200838020-00005] [PMID]

[13] Moreau D, Dubots P, Boggio V, Guilland JC, Cometti G. Effects of electromyostimulation and strength training on muscle soreness, muscle damage and sympathetic activation. Journal of Sports Sciences. 1995; 13(2):95-100. [DOI:10.1080/02640419508732216] [PMID]

[14] Vanderthommen M, Duteil S, Wary C, Raynaud JS, LeroyWillig A, Crielaard JM, et al. A comparison of voluntary and electrically induced contractions by interleaved $1 \mathrm{H}$-and 
31P-NMRS in humans. Journal of Applied Physiology. 2003; 94(3):1012-24. [DOI:10.1152/japplphysiol.00887.2001] [PMID]

[15] Paillard T. Effects of general and local fatigue on postural control: A review. Neuroscience \& Biobehavioral Reviews. 2012; 36(1):162-76. [DOI:10.1016/j.neubiorev.2011.05.009] [PMID]

[16] Paillard T, Maitre J, Chaubet V, Borel L. Stimulated and voluntary fatiguing contractions of quadriceps femoris differently disturb postural control. Neuroscience Letters. 2010; 477(1):48-51. [DOI:10.1016/j.neulet.2010.04.034] [PMID]

[17] Taylor JL, Petersen NT, Butler JE, Gandevia S. Corticospinal transmission after voluntary contractions. In: Gandevia SC, Proske U, Stuart DG, editors. Sensorimotor Control of Movement and Posture. Boston, Massachusetts: Springer; 2012. [DOI:10.1007/978-1-4615-0713-0_49]

[18] Taylor JL, Todd G, Gandevia SC. Evidence for a supraspinal contribution to human muscle fatigue. Clinical and Experimental Pharmacology and Physiology. 2006; 33(4):400-5. [DOI:10.1111/j.1440-1681.2006.04363.x] [PMID]

[19] Chaubet V, Paillard T. Effects of unilateral knee extensor muscle fatigue induced by stimulated and voluntary contractions on postural control during bipedal stance. Neurophysiologie Clinique/Clinical Neurophysiology. 2012; 42(6):377-83. [DOI:10.1016/j.neucli.2012.08.002] [PMID]

[20] Chaubet V, Maitre J, Cormery B, Paillard T. Stimulated and voluntary fatiguing contractions of quadriceps femoris similarly disturb postural control in the bipedal stance. European Journal of Applied Physiology. 2012; 112(5):1881-7. [DOI:10.1007/s00421-011-2168-9] [PMID]

[21] Dickin DC, Doan JB. Postural stability in altered and unaltered sensory environments following fatiguing exercise of lower extremity joints. Scandinavian Journal of Medicine \& Science in Sports. 2008; 18(6):765-72. [DOI:10.1111/j.16000838.2007.00760.x] [PMID]

[22] Paillard T, Chaubet V, Maitre J, Dumitrescu M, Borel L. Disturbance of contralateral unipedal postural control after stimulated and voluntary contractions of the ipsilateral limb. Neuroscience Research. 2010; 68(4):301-6. [DOI:10.1016/j. neures.2010.08.004] [PMID]

[23] Cyrus M, Akbari M, Dadgoo M, Teymuri Z. Comparing the effect of voluntary and electrical fatigue of quadriceps on postural control. Journal of Clinical Physiotherapy Research. 2016; 1(1):12-6. [DOI:10.22037/english.v1i1.8375]

[24] Tsay A, Allen TJ, Leung M, Proske U. The fall in force after exercise disturbs position sense at the human forearm. Experimental Brain Research. 2012; 222(4):415-25. [DOI:10.1007/s00221-012-3228-z] [PMID]

[25] Allen DG, Lamb GD, Westerblad H. Skeletal muscle fatigue: Cellular mechanisms. Physiological Reviews. 2008; 88(1):287-332. [DOI:10.1152/physrev.00015.2007] [PMID]

[26] Gandevia SC. Spinal and supraspinal factors in human muscle fatigue. Physiological Reviews. 2001; 81(4):1725-89. [DOI:10.1152/physrev.2001.81.4.1725] [PMID]

[27] Bigland-Ritchie BW, Woods JJ. Changes in muscle contractile properties and neural control during human muscular fatigue. Muscle \& Nerve: Official Journal of the American Association of Electrodiagnostic Medicine. 1984; 7(9):691-9. [DOI:10.1002/mus.880070902] [PMID]
[28] Hiemstra LA, Lo IK, Fowler PJ. Effect of fatigue on knee proprioception: Implications for dynamic stabilization. Journal of Orthopaedic \& Sports Physical Therapy. 2001; 31(10):598-605. [DOI:10.2519/jospt.2001.31.10.598] [PMID]

[29] Lattanzio PJ, Petrella RJ, Sproule JR, Fowler PJ. Effects of fatigue on knee proprioception. Clinical Journal of Sport Medicine: Official Journal of the Canadian Academy of Sport Medicine. 1997; 7(1):22-7. [DOI:10.1097/00042752199701000-00005]

[30] Vanderthommen M, Duteil S, Wary C, Raynaud JS, LeroyWillig A, Crielaard JM, Carlier PG. A comparison of voluntary and electrically induced contractions by interleaved $1 \mathrm{H}$-and 31P-NMRS in humans. Journal of Applied Physiology. 2003; 94(3):1012-24. [DOI:10.1152/japplphysiol.00887.2001] [PMID]

[31] Bergstrom M, Hultman E. Energy cost and fatigue during intermittent electrical stimulation of human skeletal muscle. Journal of Applied Physiology. 1988; 65(4):1500-5. [DOI:10.1152/jappl.1988.65.4.1500] [PMID]

[32] Søgaard K, Gandevia SC, Todd G, Petersen NT, Taylor JL. The effect of sustained low-intensity contractions on supraspinal fatigue in human elbow flexor muscles. The Journal of Physiology. 2006; 573(2):511-23. [DOI:10.1113/ jphysiol.2005.103598] [PMID] [PMCID]

[33] Taylor JL, Gandevia SC. A comparison of central aspects of fatigue in submaximal and maximal voluntary contractions. Journal of Applied Physiology. 2008; 104(2):542-50. [DOI:10.1152/japplphysiol.01053.2007] [PMID]

[34] Taylor JL, Petersen NT, Butler JE, Gandevia S. Corticospinal transmission after voluntary contractions. In: Gandevia SC, Proske U, Stuart DG, editors. Sensorimotor Control of Movement and Posture. Boston, Massachusetts: Springer; 2002. [DOI:10.1007/978-1-4615-0713-0_49]

[35] Henneman E, Somjen G, Carpenter DO. Functional significance of cell size in spinal motoneurons. Journal of Neurophysiology. 1965; 28(3):560-80. [DOI:10.1152/ jn.1965.28.3.560] [PMID]

[36] Ijkema-Paassen J, Gramsbergen A. Development of postural muscles and their innervation. Neural Plasticity. 2005; 12(2-3):141-51. [DOI:10.1155/NP.2005.141] [PMID] [PMCID]

[37] Massion J. Postural control system. Current Opinion in Neurobiology. 1994; 4(6):877-87. [DOI:10.1016/0959-4388(94)90137-6]

[38] Suponitsky Y, Verbitsky O, Peled E, Mizrahi J. Effect of selective fatiguing of the shank muscles on single-leg-standing sway. Journal of Electromyography and Kinesiology. 2008; 18(4):682-9. [DOI:10.1016/j.jelekin.2007.01.009] [PMID]

[39] Salavati M, Moghadam M, Ebrahimi I, Arab AM. Changes in postural stability with fatigue of lower extremity frontal and sagittal plane movers. Gait \& Posture. 2007; 26(2):214-8. [DOI:10.1016/j.gaitpost.2006.09.001] [PMID] 